\title{
ВЛИЯНИЕ НЕСТАНДАРТНЫХ ТРУДОВЫХ ГРАФИКОВ НА БАЛАНС МЕЖАУ РАБОТОЙ И ЖИЗНЬЮ (ПО ДАННЫМ ЕВРОПЕЙСКОГО СОЦИАЛЬНОГО ИССЛЕДОВАНИЯ В РОССИИ)
}

Широкое распространение нестандартных трудовых графиков (работа в вечернее и ночное время, а также в выходные дни) в контексте развития «экономики 24/7» актуализирует вопрос об оценке их влияния на качество жизни работников, в том числе на баланс между работой и другими сторонами жизни. Занятость в нестандартное время плохо вписывается в сложившиеся ритмы семейной и общественной активности, нарушая привычные паттерны социальной включенности. Это исследование представляет собой оценку масштабов и последствий нестандартных трудовых графиков в России, опираясь на данные пятого раунда «Европейского социального исследования». Около двух третей российских работников регулярно трудятся вечером, ночью и/или в выходные дни, что является одним из самых высоких показателей среди европейских стран. Результаты регрессионного анализа говорят о том, что нестандартные трудовые графики негативно сказываются на восприятии людьми баланса между работой и другими сторонами жизни. Чтобы почувствовать ухудшение баланса между работой и жизнью достаточно работать вечером/ночью несколько раз в месяц или один раз в месяц в выходные дни. Хотя результаты исследований свидетельствуют, что негативные эффекты нестандартных трудовых графиков проявляются с разной силой в зависимости от гендерных и семейных характеристик, мы не получили

Андрей Вячеславович Шевчук- к.э.н., доцент, кафедра экономической социологии, старший научный сотрудник Лаборатории экономико-социологических исследований, Национальный исследовательский университет «Высшая школа экономики», Москва, Россия. Электронная почта: shevchuk@hse.ru

Анна Валерьевна Красильникова- магистрантка программы «Прикладные методы социального анализа рынков», факультет социальных наук, Национальный исследовательский университет «Высшая школа экономики», Москва, Россия. Электронная почта: avkrasilnikova@hse.ru 
подтверждения того, что пол и наличие детей изменяют эффекты нестандартных трудовых графиков. Нестандартные трудовые графики, способные порождать серьезные социальные проблемы, заслуживают более пристального внимания научного сообщества и органов государственной власти. В частности, требуются дальнейшие исследования, включающие разнообразные индикаторы состояния здоровья, субъективного благополучия и социального самочувствия, применительно к различным категориям российских работников.

Ключевые слова: рабочее время, нестандартный график работы, баланс между работой и жизнью, Европейское социальное исследование

DOI: 10.17323/727-0634-2019-17-2-223-236

Трудовой график структурирует жизнь человека, порождая важные последствия для здоровья, социальных взаимодействий и субъективного благополучия. Поэтому исследование темпоральных режимов работы (трудовых графиков) не менее актуально, чем более традиционная проблематика продолжительности рабочего времени. В рамках индустриального общества сложился социальный стандарт занятости, подразумевающий работу пять дней в неделю с понедельника по пятницу в дневное время (условно с 9 до 17) и два выходных дня (суббота и воскресенье). Тенденция к дестандартизации и флексибилизации занятости, стремление бизнеса функционировать в режиме $24 / 7$, доступность работника вне официального рабочего времени, благодаря развитию информационно-коммуникационных технологий, ведут к широкому распространению нестандартных трудовых графиков. За пределами традиционного рабочего дня заняты не только работники непрерывных производств, экстренных служб и транспорта, но все больше- и сферы общественного питания, торговли, индустрии развлечений, контакт-центров, а также высококвалифицированные профессионалы и управленцы. Широкое распространение нестандартных графиков актуализирует вопрос об оценке их влияния на качество жизни работников и их семей. Зарубежные исследования свидетельствуют о том, что работа в нестандартное время имеет негативные последствия для здоровья и субъективного благополучия работников, обусловленные нарушением привычных биологических и социальных ритмов жизни (Iskra-Golec et al. 2016; Costa 2010; Perrucci et al. 2007; Presser 2003).

Российские исследователи пока слабо включены в разработку данной проблематики. Несмотря на то, что в отечественной науке существуют давние традиции исследования бюджетов времени, в том числе с целью анализа качества жизни в сферах труда, быта и отдыха (Караханова 2009; Патрушев и др. 2001), до сих пор неизвестны даже примерные масштабы распространения нестандартных трудовых графиков. Существуют лишь фрагментарные свидетельства о взаимосвязи трудовых графиков и субъек- 
тивного благополучия некоторых категорий российских работников. Так, отмечаются негативные аспекты нестандартных трудовых графиков в рамках гибкой и неформальной занятости в сфере услуг (Клеман 2007), повышение стресса у сотрудников контакт-центров, работающих в ночную смену (Куваева 2014), снижение удовлетворенности балансом между работой и жизнью среди фрилансеров, работающих в ночное время (Shevchuk et al. 2019).

Используя данные пятого раунда «Европейского социального исследования» (European Social Survey), мы впервые представим сравнительные данные о масштабах нестандартной занятости в России (на примере работы в вечернее и ночное время, а также в выходные дни) и оценим влияние нестандартных трудовых графиков на баланс между работой и жизнью, являющийся важным индикатором социального благополучия (Рождественская 2011).

\section{Не в такт: почему работа в нестандартное время ведет к негативным последствиям}

В зарубежной литературе накоплен богатый опыт изучения негативного влияния нестандартных трудовых графиков на жизнь работников и их семей. Можно выделить два основных направления: медицинское и социологическое; оба исходят из того, что такая работа нарушает привычные ритмы жизни.

Медицинское направление изучает влияние нестандартных трудовых графиков на физическое и психологическое здоровье человека. Сбои суточных ритмов организма, нарушение режимов сна и питания ведут к депрессивным состояниям и психосоматическим проблемам (включая головные боли, апатию, раздражительность, усталость и эмоциональное истощение), а также к желудочно-кишечным дисфункциям, заболеваниям сердечно-сосудистой системы, ухудшению репродуктивного здоровья женщин, злокачественным опухолям. Хроническая усталость и сонливость, вызванные нарушением нормальных биологических ритмов, также способствуют росту аварий на производстве и транспорте, несчастных случаев и травматизма на рабочем месте (Costa 2010; Jamal 2004).

Социологическое направление базируется на понимании темпоральной организации социальной жизни, задающей сезонные, недельные и ежедневные рутины (Lewis, Weigert 1981). Обсуждая социальную природу времени, Питирим Сорокин и Роберт Мертон обращали внимание, что «промежутки времени обретают свои особые качества благодаря связи со свойствами или действиями», в результате «количественно равные периоды времени делаются социально неравными» (Сорокин, Мертон 2004: 115-116). Социологические исследования фиксируют разную субъективную ценность времени с приоритетом вечерних часов и выходных дней, предназначенных для семейных и социальных взаимодействий 
(Arlinghaus, Nachreiner 2016). Это означает, что один час работы в конвенциональное дневное время социально неравнозначен часу работы поздним вечером или часу работы в выходные дни. Таким образом, нестандартный трудовой график не согласуется с устоявшимися ритмами социальной и семейной жизни. Человеку, работающему вечерами, ночами или в выходные дни, трудно проводить достаточное время с друзьями, знакомыми и родственниками, активно участвовать в общественных мероприятиях, разделять семейные церемонии и ритуалы, соблюдать важные для поддержания социальных отношений традиции. В результате страдает социальная включенность, нарастает ощущение конфликта между работой и другими сторонами жизни, в семьях между партнерами зреет взаимная неудовлетворенность, падает качество взаимодействия с детьми (Iskra-Golec et al. 2016; Tuttle, Garr 2012; Fenwick, Tausig 2004; Presser 2003).

Результаты многих исследований свидетельствуют, что негативные эффекты нестандартных трудовых графиков проявляются с разной силой в зависимости от гендерных и семейных характеристик. Совмещение нестандартных трудовых графиков и семейных обязанностей труднее дается женщинам, семейным парам с детьми, одиноким родителям, а также семейным парам, где оба члена семьи работают (Tuttle, Garr 2012; Costa et al. 2010; Fenwick, Tausig 2004; Presser 2003). В целом можно заключить, что в последние десятилетия эмпирические свидетельства негативного влияния нестандартных трудовых графиков на жизнь работников и их семей выявлены в исследованиях, охватывающих различные страны, сектора экономики и профессиональные группы.

\section{Нестандартные трудовые графики: Россия В международном контексте}

В большинстве репрезентативных опросов населения России содержатся данные только об общей продолжительности рабочего времени, тогда как информация о трудовых графиках отсутствует. Исключение составляет пятая волна Европейского социального исследования (ЕСИ), в рамках которой россиянам задавались вопросы о работе в вечернее и ночное время, а также в выходные дни. В России это международное исследование проводилось Институтом сравнительных социальных исследований (ЦЕССИ) в 2011 г. Исходный размер российской выборки составлял 2484 респондентов, из них 1335 имело оплачиваемую работу.

В таблице 1 представлена распространенность нестандартных трудовых графиков среди работающего населения России. Каждый второй сообщил, что работает по вечерам или ночам, по крайней мере, несколько раз в месяц, а каждый четвертый- несколько раз в неделю или ежедневно. Около $60 \%$ респондентов работают в выходные как минимум раз в месяц, а около половины- несколько раз в месяц или каждую неделю. Только 
$41 \%$ российских работников совсем не трудятся в вечернее и ночное время, а $23 \%$ - в выходные дни. При этом мужчины значительно чаще женщин работают вечером и ночью, также как и в выходные дни. Нестандартные трудовые графики шире распространены среди руководителей высшего и среднего звена, квалифицированных промышленных рабочих, работников сельского хозяйства, сферы торговли и услуг.

Таблица 1

Распространенность нестандартных трудовых графиков в России (\%)

\begin{tabular}{|c|c|c|c|c|}
\hline & & \multicolumn{2}{|c|}{ Пол } & \multirow[b]{2}{*}{$\underset{\Xi}{\Xi}$} \\
\hline & & 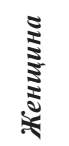 & 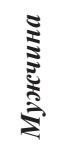 & \\
\hline \multirow{5}{*}{$\begin{array}{l}\text { Как часто на вашей работе вам } \\
\text { приходится работать } \\
\text { по вечерам или по ночам?* }\end{array}$} & Ежедневно & 3,3 & 3,4 & 3,4 \\
\hline & Несколько раз в неделю & 18,0 & 25,9 & 21,7 \\
\hline & Несколько раз в месяц & 11,6 & 21,2 & 16,2 \\
\hline & Раз в месяц и реже & 14,7 & 20,4 & 17,4 \\
\hline & Никогда & 52,4 & 29,1 & 41,3 \\
\hline \multirow{5}{*}{$\begin{array}{l}\text { Как часто на вашей работе вам } \\
\text { приходится работать по суббо- } \\
\text { там и воскресеньям? }\end{array}$} & Каждую неделю & 16,4 & 19,3 & 17,8 \\
\hline & Несколько раз в месяц & 28,7 & 33,7 & 31,1 \\
\hline & Раз в месяц & 8,6 & 11,9 & 10,1 \\
\hline & Реже, чем раз в месяц & 17,6 & 18,5 & 18,0 \\
\hline & Никогда & 28,7 & 16,7 & 23,0 \\
\hline
\end{tabular}

* В анкете использовалась семибалльная шкала, которая была перекодирована в пятибалльную.

Чтобы оценить масштабы распространения нестандартных графиков имеет смысл привести данные для всех 27 стран, входящих в пятую волну Европейского социального исследования. Сравним совокупную долю работников, которые более или менее регулярно трудятся за пределами стандартного рабочего времени. На рис. 1 представлена доля работников, которые трудятся в вечернее/ночное время несколько раз в месяц или чаще и/или в выходные дни раз в месяц или чаще.

Россия находится в группе стран с наибольшей распространенностью нестандартных трудовых графиков: $64 \%$ работников регулярно работают в нестандартное время (ночью и/или в выходные), в то время как, например, в Германии - 55\%, Франции -51\%, Нидерландах - 49\%. Очевидно, что режимы рабочего времени в разных странах тесно связаны с особенностями национальных законодательств, что требует специального рассмотрения. 
Однако уже на данном этапе можно констатировать широкое распространение нестандартных трудовых графиков во всех рассматриваемых странах (среднее по всей выборке 57\%) и особенно в России. Мы также рассчитали масштабы группы, в которой работники одновременно сталкиваются с нестандартными графиками обоих типов, т.е. обычно трудятся как в вечерние/ ночные часы (несколько раз в месяц или чаще), так и в выходные дни (раз в месяц или чаще). По этому показателю Россия также в лидирующей группе- $36 \%$ (среднее по всей выборке $30 \%$ ).

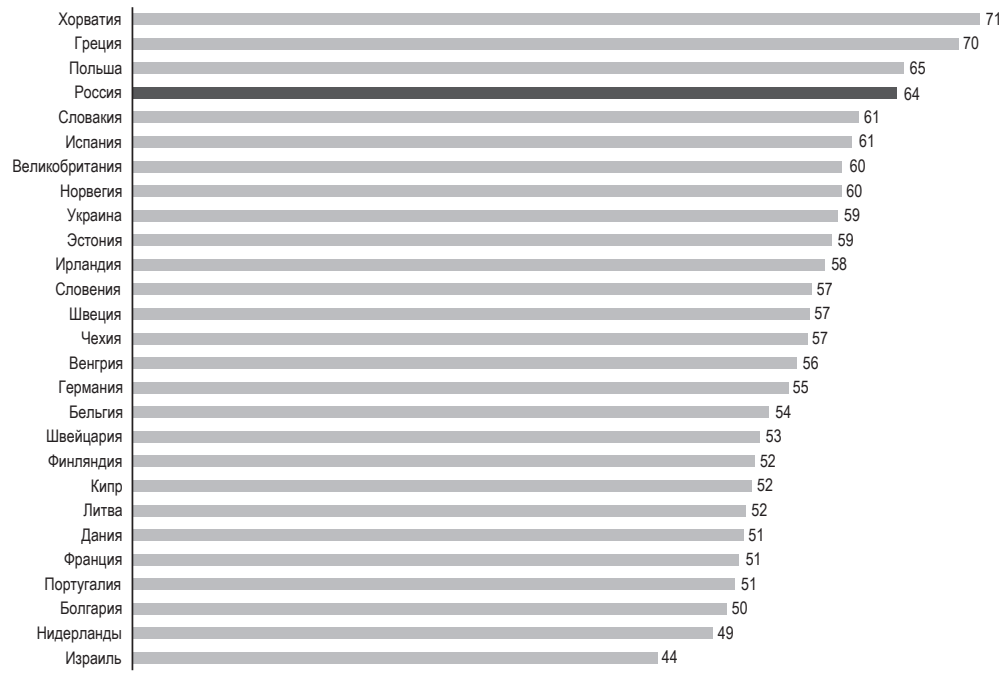

Puc. 1. Нестандартные трудовые графики в 27 странах (работа ночью и/или в выходные), \%

Следует учитывать, что респонденты ЕСИ отвечали на вопросы о трудовых графиках по основному месту работы. Учитывая, что значительная часть российских работников имеет дополнительную занятость и разного рода приработки (по некоторым оценкам, до трети работающих), реальные масштабы работы в вечерние и ночные часы, а также в выходные дни, скорее всего, недооцениваются (Покида, Зыбуновская 2017).

\section{Как нестандартный трудовой график подрывает баланс между работой и жизнью: результаты регрессионного анализа}

Мы использовали метод регрессионного анализа для оценки взаимосвязи между двумя типами нестандартного трудового графика (работа в вечернее и ночное время, работа в выходные дни) и восприятием баланса между работой и жизнью. Под «жизнью» здесь понимаются многообразные 
типы активности за пределами оплачиваемой занятости: семья/партнерство, домашний труд, культурное развитие, хобби, спорт, отдых, развлечения (Рождественская 2011). Предыдущие исследования выявили, что по сравнению с другими индикаторами субъективного благополучия (например, такими, как оценка состояния здоровья, эмоциональное выгорание, удовлетворенность жизнью в целом), работа в нестандартное время сильнее всего сказывается именно на восприятии баланса (Shevchuk et al. 2019; Greubel et al. 2016; Fenwick, Tausig 2004).

В рамках пятой волны ЕСИ респондентам предлагалось оценить по пятибалльной шкале различные аспекты, характеризующие баланс между работой и жизнью. Как часто человек чувствует себя после работы настолько усталым, что уже не можете получать удовольствие ни от чего другого, чем хотел бы заняться дома? Насколько он удовлетворен соотношением времени, которое тратит на работу, и времени, которое посвящает другим сторонам жизни? Как часто человек чувствует, что из-за работы не может посвятить семье или партнеру столько времени, сколько ему хотелось бы? Ответы респондентов на эти вопросы представляют собой их суждения, оценки, мнения, т.е. скорее субъективное восприятие действительности в отличие от объективных характеристик (например, соотношения часов, потраченных на работу и другие занятия). Поэтому в данном случае корректнее говорить именно об индивидуальном восприятии баланса между работой и жизнью. Для комплексной оценки этого явления сконструирован индекс путем вычисления среднего значения по трем вышеперечисленным пятибалльным порядковым переменным. Чем выше значение индекса, тем легче человеку поддерживать баланс работа-жизнь. Корреляции между переменными значимы на уровне $95 \%$ доверительной вероятности, коэффициент альфа Кронбаха составил 0,65. Факторный анализ методом главных компонент также показал, что три переменные попадают в один фактор примерно с равными весами. Значение индекса использовалось в качестве зависимой переменной. Мы также построили самостоятельные регрессионные модели с каждым из трех вопросов в качестве зависимой переменной и получили аналогичные результаты.

В связи с тем, что при ответе на третий вопрос 265 респондентов выбрали вариант, что они не имеют семьи или партнера, мы были вынуждены исключить их из анализируемой выборки. В результате остались только те, у кого есть семья или партнер (респонденты могут проживать вместе с партнером или раздельно). С одной стороны, это ограничение исследования, с другой-мы можем более полно оценить влияние нестандартных графиков работы на субъективное благополучие людей, включенных в более широкий круг социальных взаимодействий, и тем самым более полно продемонстрировать социальные последствия нестандартных трудовых графиков. Мы также удалили из анализируемой выборки 21 человека, работающего менее 20 часов в неделю. Итоговая выборка составила 1049 человек. 
Независимыми переменными являлись занятость в вечернее или ночное время и занятость в выходные дни. Данные переменные перекодированы в дихотомические (каждая категория преобразована в отдельную переменную со значениями 1 и 0). Регрессионный анализ позволяет выявить наличие или отсутствие взаимосвязи между трудовыми графиками и субъективным благополучием «при прочих равных», т.е. предполагая, что все остальные контрольные переменные, включенные в регрессионную модель, принимают одни и те же значения, и тем самым их возможное влияние на ощущение баланса между работой и жизнью нивелируется. В качестве контрольных использованы дихотомические переменные (пол, семейное положение, наличие детей до 16 лет), интервальные (возраст, количество лет образования, количество рабочих часов в неделю) и порядковые, перекодированные в дихотомические (профессия по классификации ISCO, уровень дохода домохозяйства). Важно отметить, что мы контролируем общую продолжительность рабочего времени, т.е. анализируем чистый эффект работы в нестандартное время независимо от количества рабочих часов в неделю. Несмотря на то, что в регрессионных моделях использовался широкий спектр контрольных переменных, данные ЕСИ (как и любые другие) имеют ограничения и не содержат всех возможных переменных, которые могут оказывать влияние на социальное самочувствие работников, что следует учитывать при интерпретации результатов данного исследования. Анализ количественных данных проведен с помощью статистического пакета SPSS. Основным методом анализа является линейная регрессия, так как зависимая переменная- интервальная.

\section{Занятость в вечернее и ночное время}

В результате построения линейной регрессии нестандартизованные коэффициенты оказались значимы для трех переменных, касающихся занятости в вечернее и ночное время: занятость вечером/ночью ежедневно, несколько раз в неделю, несколько раз в месяц оказывает значимый негативный эффект на восприятие баланса между работой и жизнью (табл. 2). Для того чтобы понять, различается ли сила эффекта в зависимости от частоты занятости, построены четыре регрессии с последовательной заменой референтной группы (в базовой регрессии референтной группой было отсутствие у работника опыта занятости в вечерние или ночные часы). В результате есть основания полагать, что частая занятость в ночное время (несколько раз в месяц и чаще) имеет значимо более высокие отрицательные показатели индекса, чем редкая занятость (раз в месяц и реже). Причем если человек занят ежедневно в ночное время, то ему значительно труднее выделять время на семью и иные стороны своей жизни, чем людям, которые работают не так часто. Работники, занятые ночью несколько раз в неделю значимо отличаются от остальных групп, как и люди, работающие несколько раз в месяц. То есть чем чаще человек 
занят в ночное время, тем труднее ему поддерживать баланс между работой и жизнью.

Таблица 2

Коэффициенты линейной регрессии

\begin{tabular}{|c|c|c|c|c|c|}
\hline & & \multicolumn{4}{|c|}{ Индекс баланса работа-жнизь } \\
\hline & & \multicolumn{2}{|c|}{$\begin{array}{c}\text { Модель } 1 \\
\text { независ. пер. } \\
\text { "занятость } \\
\text { в вечернее, ночное } \\
\text { время" }\end{array}$} & \multicolumn{2}{|c|}{$\begin{array}{c}\text { Модель } 2 \\
\text { независ. пер. «заня- } \\
\text { тость } \\
\text { в выходные дни» }\end{array}$} \\
\hline & & $\boldsymbol{B}$ & S.E. & $\boldsymbol{B}$ & S.E. \\
\hline $\begin{array}{l}\text { Занятость } \\
\text { в вечернее, } \\
\text { ночное время }\end{array}$ & $\begin{array}{l}\text { ежедневно } \\
\text { несколько раз } \\
\text { в неделю } \\
\text { несколько раз } \\
\text { в месяц } \\
\text { раз в месяц и реже }\end{array}$ & $\begin{array}{l}-0,895 \\
-0,425 \\
-0,217 \\
-0,139\end{array}$ & $\begin{array}{r}0,192^{* * *} \\
0,085^{* * *} \\
0,096^{*} \\
0,087\end{array}$ & & \\
\hline $\begin{array}{l}\text { Занятость } \\
\text { в выходные дни }\end{array}$ & $\begin{array}{l}\text { каждую неделю } \\
\text { несколько раз } \\
\text { в месяц } \\
\text { раз в месяц } \\
\text { реже, чем } \\
\text { раз в месяц }\end{array}$ & & & $\begin{array}{l}-0,412 \\
-0,356 \\
-0,514 \\
-0,034\end{array}$ & $\begin{array}{r}0,104^{* * *} \\
0,087^{* * *} \\
0,114^{* * *} \\
0,093\end{array}$ \\
\hline \multicolumn{2}{|c|}{ Пол (мужчины- реф.) } & $-0,025$ & 0,076 & $-0,013$ & 0,077 \\
\hline \multicolumn{2}{|c|}{$\begin{array}{l}\text { Семейное положение (без партнера- } \\
\text { реф.) }\end{array}$} & 0,117 & 0,075 & 0,100 & 0,076 \\
\hline \multicolumn{2}{|c|}{$\begin{array}{l}\text { Наличие детей до } 16 \text { лет (отсутствие } \\
\text { детей-реф.) }\end{array}$} & $-0,149$ & $0,066^{*}$ & $-0,188$ & $0,066^{* *}$ \\
\hline \multicolumn{2}{|l|}{ Константа } & & *** & & *** \\
\hline \multicolumn{2}{|c|}{$\mathrm{R}^{2}$ /скорректированный $\mathrm{R}^{2}$} & \multicolumn{2}{|c|}{$0,124 / 0,103$} & \multicolumn{2}{|c|}{$0,121 / 0,099$} \\
\hline \multicolumn{2}{|c|}{$\mathrm{F}$ для изменения $\mathrm{R}^{2}$} & \multicolumn{2}{|r|}{$5,893^{* * *}$} & \multicolumn{2}{|r|}{$5,693^{* * *}$} \\
\hline
\end{tabular}

Примечание. ${ }^{* * *} \mathrm{p}<0.001,{ }^{* *} \mathrm{p}<0.01,{ }^{*} \mathrm{p}<0.05$. Остальные контрольные переменные в таблице не показаны.

\section{Занятость в выходные дни}

Нестандартизованные регрессионные коэффициенты оказались значимыми для трех переменных, касающихся занятости в выходные дни: занятость в выходные каждую неделю, несколько раз в месяц и раз в месяц отрицательно влияет на возможность поддержания баланса работа-жизнь. 
Регрессии с заменой референтной группы показали, что негативный эффект от частой занятости в выходные дни (каждую неделю, несколько раз в месяц, раз в месяц) значимо выше, чем от редкой занятости (реже одного раза в месяц). При этом нет статистически значимых различий между эффектами занятости в выходные дни каждую неделю, несколько раз в месяц и раз в месяц. То есть если человек работает в выходные дни как минимум раз в месяц, он будет испытывать ухудшение баланса между работой и жизнью (неважно, будет ли он работать раз в месяц или несколько раз в неделю, эффект будет одинаковым).

Важным является вопрос о том, насколько универсальными являются последствия нестандартных графиков или, другими словами, существуют ли наиболее уязвимые группы. Для решения этой задачи построены модели с эффектами взаимодействия между занятостью в нестандартное время и полом, а также занятостью и наличием детей до 16 лет. Переменные, связанные с трудовой деятельностью, перекодированы в дихотомические: если человек работал ночью несколько раз в месяц и чаще, то присваивалось значение 1, если реже, то 0; если человек работал в выходные дни раз в месяц и чаще, то присваивалось значение 1 , если реже, то 0 . Такое разделение основывается на предыдущем анализе: баланс работа/жизнь одинаково сложно поддерживать в случае, когда человек работает в нестандартное время редко или вовсе занят только в стандартные часы. Переменные пол и наличие детей также являются дихотомическими. Эффект взаимодействия был сконструирован путем перемножения двух переменных: занятости и социально-демографической характеристики. В результате мы не получили подтверждения того, что пол и наличие детей изменяют эффект ночного рабочего времени или выходного дня ${ }^{1}$.

Хотя в данном исследовании негативные эффекты графиков не зависят от пола и наличия детей, это не означает, что эти эффекты абсолютно универсальны, т.к. возможны другие, в том числе более сложные сочетания индивидуальных характеристик. Например, в исследовании русскоязычных фрилансеров выявлен значимый эффект пола в сочетании с наличием партнера (Shevchuk et al. 2019). Это же исследование ставит интересный вопрос о сравнении наемных и самозанятых работников. Небольшой размер выборки не позволил нам построить более разнообразные и сложные регрессионные модели.

\section{Заключение}

В современном обществе нарастает конфликт между стремлением бизнеса функционировать в режиме 24/7, поддерживаемого развитием «общества потребления» и более инерционной темпоральной организацией

\footnotetext{
${ }^{1}$ Результаты предоставляются авторами по запросу.
} 
социальной и семейной жизни. Для обеспечения доступности разнообразных благ в любое время суток на протяжении недели все больше людей вынуждены работать в вечерние и ночные часы, в выходные и праздничные дни, предназначенные для времяпрепровождения с родственниками, друзьями, знакомыми, а также разнообразных общественных мероприятий. В результате занятые в нестандартное время оказываются исключенными из привычной социальной активности, что отрицательно сказывается на их субъективном благополучии.

Настоящее исследование представляет собой первую попытку оценить масштабы и последствия нестандартных режимов работы в России. В группе риска находятся почти две трети российских работников, которые регулярно трудятся в нестандартное время. Согласно результатам нашего анализа, чтобы почувствовать ухудшение баланса между работой и жизнью достаточно работать вечером/ночью несколько раз в месяц или же хотя бы один раз в месяц в выходные дни. При этом, учитывая распространенность дополнительной занятости, есть основания полагать, что масштабы нестандартных режимов работы недооцениваются. Требуются дальнейшие исследования для более глубокого понимания последствий нестандартных трудовых графиков, включая разнообразные индикаторы состояния здоровья, субъективного благополучия и социального самочувствия, применительно к различным категориям работников. Специального внимания заслуживает анализ проблемы в контексте неформальной занятости, когда работа в нестандартное время выпадает из-под защиты трудового законодательства, еще более ухудшая положение работника. Можно заключить, что широкое распространение нестандартных трудовых графиков в России имеет серьезные социальные последствия, которые заслуживают более пристального внимания научного сообщества и органов государственной власти.

\section{Выражения признательности}

Исследование осуществлено в рамках Программы фундаментальных исследований НИУ ВШЭ в 2019 г.

\section{Список источников}

Караханова Т. М. (2009) Время работающих горожан: тенденции использования в 1986-2008 годы. Социологический журнал, (2): 68-81.

Клеман К. (2007) «Флексибильность по-российски»: очерк о новых формах труда и подчинения в сфере услуг. Социологический журнал, (4): 74-96.

Куваева И.О. (2014) Особенности профессионального стресса у сотрудников контактцентров в зависимости от графика работы. Известия Уральского федерального университета. Сер. 1, Проблемы образования, науки и культуры, 123 (1): 183-188. 
Патрушев В. Д., Артемов В.А., Новохацкая О. В. (2001) Изучение бюджетов времени в России XX в. Сочиологические исследования, (6): 112-120.

Покида А.Н., Зыбуновская Н.В. (2017). О тенденциях занятости работающего населения. Социологические исследования, (7):54-59.

Рождественская Е.Ю. (2011) Концепция баланса жизни и труда: уроки европейской социальной политики и российские перспективы. Журнал исследований социильной политики, 9 (4): 439-454.

Сорокин П., Мертон Р. (2004) Социальное время: опыт методологического и функционального анализа. Соииологические исследования, (6): 112-119.

Arlinghaus A., Nachreiner F. (2016) Unusual and Unsocial? Effects of Shift Work and Other Unusual Working Times on Social Participation. In: I. Iskra-Golec, J. Barnes-Farrell, P. Bohle (eds.) Social and Family Issues in Shift Work and Non Standard Working Hours. Cham: Springer:39-57.

Costa G. (2010) Shift Work and Health: Current Problems and Preventive Actions. Safety and Health at Work, 1 (2): 112-123.

Fenwick R., Tausig M. (2004) The Health and Family-Social Consequences of Shift Work and Schedule Control: 1977 and 1997. In: C.F. Epstein, A.L. Kalleberg (eds.) Fighting For Time: Shifting Boundaries of Work and Social Life. New York: Russell Sage Foundation:77-110.

Greubel J., Arlinghaus A., Nachreiner F., Lombardi D. (2016) Higher Risks when Working Unusual Times? A Cross-validation of the Effects on Safety, Health, and Work-life Balance. International archives of occupational and environmental health, 89 (8): 1205-1214.

Iskra-Golec I., Barnes-Farrell J., Bohle P. (2016) Social and Family Issues in Shift Work and Non Standard Working Hours. Cham: Springer.

Jamal M. (2004) Burnout, Stress and Health of Employees on Non-standard Work Schedules: a Study Of Canadian Workers. Stress and Health, 20 (3): 113-119.

Lewis J. D., Weigert A. J. (1981) The Structures and Meanings of Social Time. Social Forces, 60 (2): 432-462.

Perrucci R., MacDermid S., King E., Tang C., Brimeyer T., Ramadoss K., Kiser S., Swanberg J. (2007) The Significance of Shift Work: Current Status and Future Directions. Journal of Family and Economic Issues, 28 (4): 600-617.

Presser H. (2003) Working in a 24/7 Economy: Challenges for American Families. New York: Russell Sage Foundation.

Shevchuk A., Strebkov D., Davis S. (2019) The Autonomy Paradox: How Night Work Undermines Subjective Well-Being of Internet-Based Freelancers. Industrial and Labor Relations Review, 72 (1): 75-100.

Tuttle R., Garr M. (2012) Shift Work and Work to Family Fit: Does Schedule Control Matter? Journal of Family and Economic Issues, 33 (3):261-271. 
Andrey Shevchuk, Anna Krasilnikova

\title{
THE IMPACT OF NON-STANDARD WORK SCHEDULES ON THE WORK-LIFE BALANCE: THE CASE OF THE EUROPEAN SOCIAL SURVEY IN RUSSIA
}

\begin{abstract}
Work schedule structures individual life and matches biological and social rhythms in various ways. Given the widespread prevalence of nonstandard work schedules (such as working evenings, nights and weekends) in the context of the '24/7 economy', the need for research into how these schedules affect worker well-being, including the issues of work-life balance, has never been greater. It is difficult for a person working in evenings, nights or weekends to spend enough time with friends, acquaintances and relatives, actively participate in social events, share family ceremonies and rituals, and keep up the traditions that are important for maintaining social relations. This study is the first attempt to estimate the occurrence and effects of nonstandard work schedules in Russia, using the data of European Social Survey ( $5^{\text {th }}$ round). About twothirds of Russian workers work evenings, nights or weekends and this is one of the highest figures among European countries. Regression models reveal that nonstandard work schedules have negative effects on perceived work-life balance. Indeed, it appears that working several times a month or even once a month on weekends in the evening or at night is enough to bring about a deterioration in the work-life balance. Although the results of many studies suggest that the negative effects of non-standard work schedules manifest themselves differently depending on gender and family characteristics, in this study we did not confirm that gender and presence of children moderate the effects of non-standard work schedules on work-life balance. The academic community and government bodies should pay more attention to nonstandard work schedules, which can generate serious social problems. Further research should include a wider variety of indicators of health and subjective well-being, as applied to various categories of Russian workers.
\end{abstract}

Key words: working time, non-standard work schedules, work-life balance, European Social Survey

DOI: $10.17323 / 727-0634-2019-17-2-223-236$

\section{References}

Arlinghaus A., Nachreiner F. (2016) Unusual and Unsocial? Effects of Shift Work and Other Unusual Working Times on Social Participation. In: I. Iskra-Golec, J. Barnes-Farrell, P. Bohle (eds.) Social and Family Issues in Shift Work and Non Standard Working Hours. Cham: Springer:39-57.

Andrey Shevchuk-Candidate of Sciences (PhD) in Economic Theory, Associate Professor of Department of Economic Sociology, Senior Research Fellow of Laboratory for Studies in Economic sociology, National Research University Higher School of Economics, Moscow, Russian Federation. Email: shevchuk@hse.ru

Anna Krasilnikova-student of the master's Programme 'Applied Methods of Social Analysis of Markets', Faculty of Social Sciences, National Research University Higher School of Economics, Moscow, Russian Federation. Email: avkrasilnikova@hse.ru 
Costa G. (2010) Shift Work and Health: Current Problems and Preventive Actions. Safety and Health at Work, 1 (2): 112-123.

Fenwick R., Tausig M. (2004) The Health and Family-Social Consequences of Shift Work and Schedule Control: 1977 and 1997. In: C. F. Epstein, A. L. Kalleberg (eds.). Fighting For Time: Shifting Boundaries of Work and Social Life. New York: Russell Sage Foundation: 77-110.

Greubel J., Arlinghaus A., Nachreiner F., Lombardi, D. (2016) Higher Risks when Working Unusual Times? A Cross-validation of the Effects on Safety, Health, and Work-life Balance. International Archives of Occupational and Environmental Health, 89 (8): 1205-1214.

Iskra-Golec I., Barnes-Farrell J., Bohle P. (2016) Social and Family Issues in Shift Work and Non Standard Working Hours. Cham: Springer.

Jamal M. (2004) Burnout, Stress and Health of Employees on Non-standard Work Schedules: A Study of Canadian Workers. Stress and Health, 20 (3): 113-119.

Karakhanova T.M. (2009) Vremya rabotayushchikh gorozhan: tendentsii ispol'zovaniya v 19862008 gody [The Time of Working Citizens: Usage Trends in 1986-2008]. Sotsiologicheskiy zhurnal [Sociological Journal], (2): 68-81.

Kleman K. (2007) 'Fleksibil'nost' po-rossiyski': ocherk o novykh formakh truda i podchineniya v sfere uslug ['Russian Flexibility': An Essay about New Forms of Labor and Subordination in Service Sector]. Sotsiologicheskiy zhurnal [Sociological Journal], (4): 74-96.

Kuvaeva I. O. (2014) Osobennosti professional'nogo stressa u sotrudnikov kontakt-tsentrov v zavisimosti ot grafika raboty [Features of Professional Stress of Employees in Contact Centres Depending on Work Schedule]. Izvestiya Ural'skogo federal'nogo universiteta. Ser. 1, Problemy obrazovaniya, nauki i kul'tury [Izvestia Ural Federal University Journal. Series 1. Issues in Education, Science and Culture], 123 (1): 183-188.

Lewis J.D., Weigert A. J. (1981) The Structures and Meanings of Social Time. Social Forces, 60 (2): 432-462.

Patrushev V.D., Artemov V.A., Novokhatskaya O. V. (2001) Izuchenie byudzhetov vremeni v Rossii XX v [The Study of Time Budgets in Twentieth Century Russia]. Sotsiologicheskie issledovaniya [Sociological Studies], (6): 112-120.

Pokida A. N., Zybunovskaya N. V. (2017). O tendentsiyakh zanyatosti rabotayushchego naseleniya [About Employment Trends in the Working Population]. Sotsiologicheskie issledovaniya [Sociological Studies], (7): 54-59.

Perrucci R., MacDermid S., King E., Tang C., Brimeyer T., Ramadoss K., Kiser S., Swanberg J. (2007). The Significance of Shift Work: Current Status and Future Directions. Journal of Family and Economic Issues, 28 (4): 600-617.

Presser H. (2003) Working in a 24/7 Economy: Challenges for American Families. New York: Russell Sage Foundation.

Rozhdestvenskaya E. Yu. (2011) Kontseptsiya balansa zhizni i truda: uroki evropeyskoy sotsial'noy politiki i rossiyskie perspektivy [The Concept of Work-Life Balance: the Lessons of European Social Policy and Russian Perspectives]. Zhurnal issledovaniy sotsial'noy politiki [The Journal of Social Policy Studies], (9) 4:439-454.

Shevchuk A., Strebkov D., Davis S. (2019) The Autonomy Paradox: How Night Work Undermines Subjective Well-Being of Internet-Based Freelancers. Industrial and Labor Relations Review, 72 (1): $75-100$.

Sorokin P., Merton R. (2004) Sotsial'noe vremya: opyt metodologicheskogo i funktsional'nogo analiza [Social Time: A Methodological and Functional Analysis]. Sotsiologicheskie issledovaniya [Sociological Studies], (6): 112-119.

Tuttle R., Garr M. (2012) Shift Work and Work to Family Fit: Does Schedule Control Matter? Journal of Family and Economic Issues, 33 (3): 261-271. 